\title{
Ultrafast Laser Fabrication of Bragg Waveguides in GLS Chalcogenide Glass
}

\author{
Ben McMillen, Botao Zhang, and Kevin Chen* \\ Department of Electrical and Computer Engineering, University of Pittsburgh, Pittsburgh, PA, 15213 \\ *Email: pec9@pitt.edu
}

\begin{abstract}
We present work on the fabrication of Bragg waveguides in gallium-lanthanum-sulfide chalcogenide glass using an ultrafast laser. Waveguides were written with a single pass while modulating the writing beam. The spatial and temporal profile of the writing beam was controlled during waveguide fabrication in order to control the shape and size of the waveguide cross-section.
\end{abstract}

Over the last decade, ultrafast lasers have become the staple research tool of many research institutions. The applications of these lasers have moved beyond basic materials studies toward ever more complex systems, such as micro-fluidic devices [1-2], micromechanical actuators [3], and integrated monolithic waveguide lasers [4-5]. The materials used in these studies are often silica-based glasses, however considerable effort has been put toward the study of highly non-linear optical materials such as chalcogenide glass [6].

Waveguide fabrication in highly nonlinear, high-index materials presents significant challenges for ultrafast laser processing. High nonlinearities contribute to temporal distortion of focused femtosecond pulses, depleting pulse energy and distorting the focal volume, while a high index contrast further contributes to spatial distortions due to index mismatch induced spherical aberration. Building on previous work [7], we present the application of both spatial beam shaping and temporal pulse tailoring for the fabrication of Bragg waveguides in gallium-lanthanum-sulfide (GLS) chalcogenide glass.

The waveguides presented here were fabricated using a Ti:sapphire system, delivering 150 pulses at $800 \mathrm{~nm}$ and a repetition rate of $250 \mathrm{kHz}$. The GLS sample was mounted to a motion stage system, incorporating a fixed focusing objective with a numerical aperture of 0.75 . Distortions due to index-mismatch induced spherical aberrations were corrected at the focal volume with a cylindrical de-magnifying telescope, providing a demagnification factor of 3 in the y direction (perpendicular to the writing direction). Finally, the pulse-compressor of the laser was adjusted to provide a pulse duration of $\sim 1.5$ picoseconds, which was has been shown to give the lowest loss waveguides in GLS chalcogenide [7].
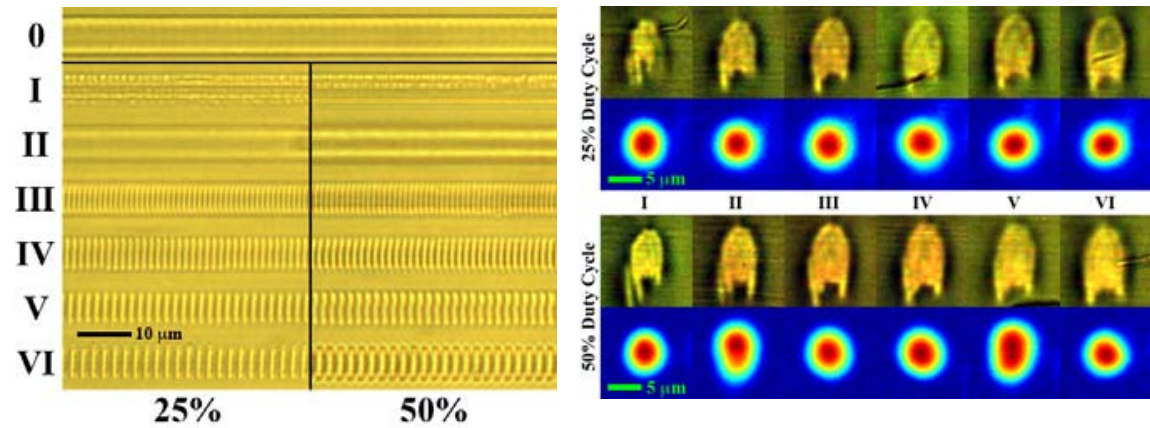

Figure 1: (a) Top-view of laser-written Bragg waveguides showing both $25 \%$ and $50 \%$ duty cycle for $1^{\text {st }}$ through $6^{\text {th }}$ order. A $0^{\text {th }}$ order (no grating) waveguide is shown at the top of the image for comparison. (b) End-fact view of the guiding structures for all orders along with a false-color guide mode image.

Figure 1 shows a top view (left) and end facet view (right) for a series of Bragg waveguides written with a pulse energy of $300 \mathrm{~nJ}$ and a fixed writing depth of $\sim 275$ microns below the material surface. This depth was chosen to preserve end facet quality after polishing, as the edges of the GLS sample were prone to chipping from regular handling during characterization. Modulation of the writing beam was controlled by supplying an external signal to the cavity dumper of the laser system at a frequency based on a fixed velocity of $1 \mathrm{~mm} / \mathrm{s}$ in order to give the desired grating period. First through sixth order Bragg waveguides were written, with a zero-order (no grating) waveguide written for comparison.

Here multi-order gratings, in combination with a high-magnification writing objective and variable duty cycle of the beam modulation, were chosen in order to determine the relationship between the grating quality (spectral) and the achievable grating resolution within the GLS sample. Gratings were designed for a center wavelength of $1550 \mathrm{~nm}$ for testing and interrogation purposes, however calculations show that for an effective index of $\sim 2.37$ in GLS chalcogenide, the spot size of the focused beam will exceed the required grating period of $\sim 329.5 \mathrm{~nm}$. This is apparent when examining the top-view of the first order grating in Figure 1, as no grating is visible and the waveguide structure appears rough and non-uniform (as compared to the un-modified waveguide, top). Grating

This is an Open Access article distributed under the terms of the Creative Commons Attribution License 2.0, which permits unrestricted use, distribution, and reproduction in any medium, provided the original work is properly cited. 
structure begins to appear at second order and above, though the image-capture resolution of the microscope system used for analysis was insufficient to capture this detail for the second-order case.

As the grating order is increased, the individual grating periods begin to appear non-uniform, and the morphology changes noticeably from one grating set to the next. This is most evident for grating orders four through six, where the shape of each period changes dramatically over the width of the waveguide. It was also found that the directionality of the non-uniform periods demonstrated a relation to writing direction, which for this experiment was from right to left in Figure 1.

We believe there are several possible causes for this observation. The first is that the use of a high-magnification writing objective, while providing increased writing resolution within the GLS substrate, has the disadvantage of increasing the local intensity within the focal volume. Higher focal intensity combined with large nonlinearities present in the GLS substrate likely lead to self-focusing and filamentation [8]. This is most evident in the end-facet view of Figure 1 (right), where extra 'tails' are found for each waveguide, as well as the presence of separated filamentation zones for the first-order waveguide. In addition to these effects, the cross-sectional size of the waveguide was strongly dependent on the laser fluence at different duty cycles. A change in grating period width with duty cycle was expected, however the lateral extent of the waveguide in the z-direction also increased for $50 \%$ duty cycle, often leading to multi-mode guiding, as evident in Figure 1 (bottom right). These results suggest that thermal effects also play a role in waveguide formation in chalcogenide glass.
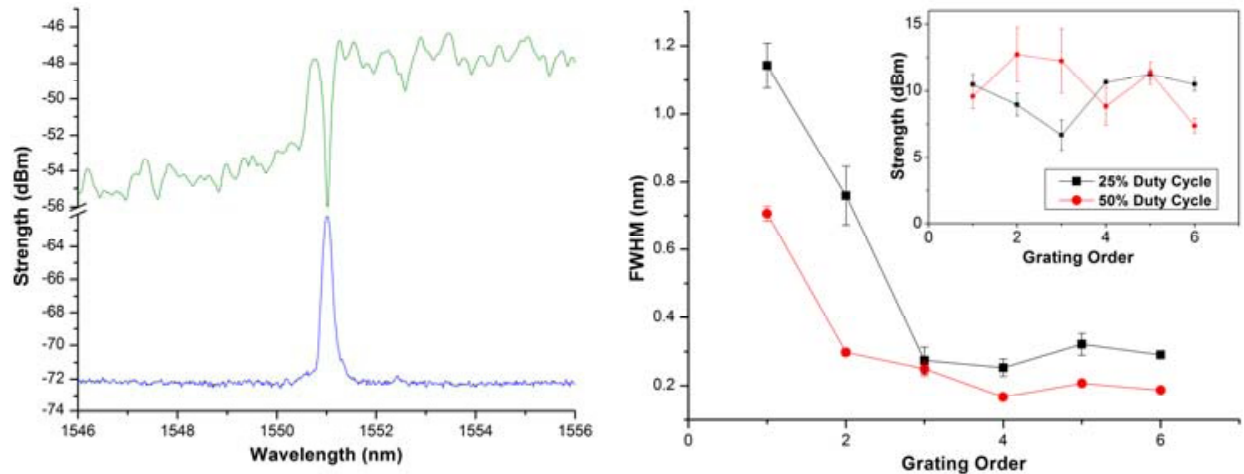

Figure 2: Spectral response of a $20 \mathrm{~mm}$ long fourth order grating written with $25 \%$ duty cycle (left) showing reflection (blue) and transmission (green) spectra. The overall line width of the gratings improves with increasing grating order (right). Inset shows grating peak as a function of grating order for the reflected spectrum.

Spectral response of the Bragg waveguides generally improved with increasing grating order, as shown in Figure 2 (right), owing to the increase in index contrast as the grating period separation becomes larger. We estimate the index contrast of the gratings to be $\sim 1 \times 10^{-3}$ [7]. Figure 2 (left) shows grating spectra for a $20 \mathrm{~mm}$ long $4^{\text {th }}$ order grating with a reflectivity of $\sim 88.13 \%$. Generally, reflectivity was found to vary widely over several trials of the same grating order (all written in the same sample). We believe this was mainly due to the difficulty in obtaining a high-quality polish on the end facets of the sample combined with variations in coupling during interrogation.

These results show promise for the fabrication of advanced devices in highly nonlinear materials, however more work is required to optimize the process to reduce losses and balance index contrast, grating quality, and spectral response.

[1] F. Bragheri, P. Minzioni, R. Martinez Vazquez, N. Bellini, P. Paiè, C. Mondello, R. Ramponi, I. Cristiani, and R. Osellame, "Optofluidic integrated cell sorter fabricated by femtosecond lasers," Lab Chip 12, 3779-3784 (2012).

[2] F. He, Y. Cheng, Z. Xu, Y. Liao, J. Xu, H. Sun, C. Wang, Z. Zhou, K. Sugioka, K. Midorikawa, Y. Xu, and X. Chen, "Direct fabrication of homogeneous microfluidic channels embedded in fused silica using a femtosecond laser," Opt. Lett. 35, 282-284 (2010).

[3] B. Lenssen and Y. Bellouard. "Optically transparent glass micro-actuator fabricated by femtosecond laser exposure and chemical etching", Appl. Phys. Lett. 101, 103503 (2012).

[4] Ams: M. Ams, P. Dekker, G. Marshall, and M. Withford, "Ultrafast laser-written dual-wavelength waveguide laser," Opt. Lett. 37, 993995 (2012).

[5] Yang Tan, Feng Chen, J. R. Vazquez de Aldana, G. A. Torchia, A. Benayas, and D. Jaque, “Continuous wave laser generation at $1064 \mathrm{~nm}$ in femtosecond laser inscribed Nd:YVO 4 channel waveguides", Appl. Phys. Lett. 97, 031119 (2010).

[6] B. Eggleton, B. Luther-Davies and K. Richardson, "Chalcogenide Photonics," Nature Photonics 5, 141-148, (2011).

[7] B. McMillen, B. Zhang, K. Chen, A. Benayas, and D. Jaque, "Ultrafast laser fabrication of low-loss waveguides in chalcogenide glass with $0.65 \mathrm{~dB} / \mathrm{cm}$ loss," Opt. Lett. 37, 1418-1420 (2012).

[8] A. Ferrer, A. Ruiz de la Cruz, D. Puerto, W. Gawelda, J. VallÈs, M. Rebolledo, V. Berdejo, J. Siegel, and J. Solis, "In situ assessment and minimization of nonlinear propagation effects for femtosecond-laser waveguide writing in dielectrics," J. Opt. Soc. Am. B 27, 1688-1692 (2010). 